\title{
Acoustic rhinometric evaluation of the nasal cavity after rapid maxillary expansion
}

\author{
Ribeiro, Annelise Nazareth Cunha *; Rino-Neto, José *; Paiva, Joáo Batista de * \\ * Department of orthodontics University of São Paulo, São Paulo, Brazil
}

\begin{abstract}
Objective: Because of the anatomic proximity between the nasal cavity and the maxilla, much has been investigated regarding changes in nasal geometry after this procedure. In this study, we propose to evaluate the repercussion of RME in the nasal cavity in the patient during the growth phase.

Materials and methods: For this, we evaluated 19 patients with transverse maxillary deficiency and indication for RME. The patients were evaluated using acoustic rhinometry in 3 moments (pre-RME, post-RME, post-restraint).

Results: There was no mean change in MCA1 M1, M2 and M3 ( $\mathrm{p}=0.122)$. MCA2 measurement appears to increase in M2. VOL 1 is suffering a mean increase in M2 compared to M1 $(\mathrm{p}=0.025)$ and continues higher in M3 ( $\mathrm{p}=0.271)$. There is little variation of VOL 2 between the evaluated moments.

Conclusion: The results allow us to affirm that RME significantly increases the anterior region of the nasal cavity immediately to the procedure, however, after the period of containment there is a tendency of recurrence of this increase returning to values close to the initial cross-sectional area of the nasal cavity.

Nazareth Cunha Ribeiro A, Rino-Neto J, Batista de Paiva J. Acoustic Rhinometric Evaluation of the Nasal Cavity After Rapid Maxillary Expansion. South Eur J Orthod Dentofac Res. 2018;5(1):6-11.
\end{abstract}

Submitted: December 20, 2017; Revised: March 15, 2018; Published: April 30, 2018

\section{INTRODUCTION}

The nasal breath is the only one considered normal and physiological because it is present at birth. However, there may be transference to the predominantly oral respiratory pattern when there is nasal obstruction, which may lead to alterations in the development of dentofacial structures. ${ }^{1}$

Many authors ${ }^{1-4}$,consider that oral breathing is one of the etiological factors of transverse maxillary deficiency. However, this cause-effect relationship continues to be much discussed due to controversies in the literature. ${ }^{5,6}$

Rapid maxillary expansion (RME) is an excellent method for the correction of transverse maxillary deficiency through the opening of the palatine suture. ${ }^{7-11}$ Due to the anatomical proximity between the nasal cavity and the maxilla, much has been investigated regarding the changes in nasal geometry after this procedure (RME). Some authors ${ }^{9,12-14}$ have demonstrated

Corresponding Author:

Ribeiro Annelise Nazareth Cunha

Department of orthodontics

University of São Paulo,

Sáo Paulo, Brazil

e-mail:annelisencr@gmail.com that the benefits of this procedure in patients during the growth phase go beyond the correction of malocclusion and may have repercussions in the nasal cavity.

Some researchers ${ }^{11,12,14,15-17}$ have associated RME with a reduction in nasal respiratory resistance, increased airflow, increased nasal cavity size and a change in respiratory mode from oral to nasal. However, other studies did not observe significant changes in the nasal cavity after $\mathrm{RME}^{18,19}$, so this subject is not yet fully clarified in the literature, thus requiring additional studies.

Acoustic rhinometry was introduced by Hilberg et al. ${ }^{20}$ and has been described as an accurate and reproducible method of evaluation of the anterior nasal cavity, being considered an objective, easily performed, non-invasive and well-tolerated examination by adults or children. ${ }^{21,22}$ When compared with other methods of evaluation such as computed tomography, rhinomanometry and magnetic resonance, the results obtained with acoustic rhinometry are equivalent in determining the nasal geometry of the anterior portion of the nasal cavity. ${ }^{23,24,25}$ In this study we proposed to evaluate the alterations in the nasal cavity, using acoustic rhinometry, of patients with transverse maxillary deficiency before, immediately after and 6 months after Rapid Maxillary Expansion (RME) treatment. 
Table 1. Distribution of age and gender.

\begin{tabular}{lcc}
\hline Patient & Age & Gender \\
\hline 1. BCF & 7 & $\mathrm{~F}$ \\
\hline 2. CVSL & 9.5 & $\mathrm{M}$ \\
\hline 3. DHB & 8.6 & $\mathrm{M}$ \\
\hline 4. FCL & 7.5 & $\mathrm{~F}$ \\
\hline 5. GSP & 9.4 & $\mathrm{M}$ \\
\hline 6. LMP & 7.6 & $\mathrm{~F}$ \\
\hline 7. LRA & 12.5 & $\mathrm{M}$ \\
\hline 8. MPFM & 9.3 & $\mathrm{M}$ \\
\hline 9. MFS & 9 & $\mathrm{M}$ \\
\hline 10. MCS & 7 & $\mathrm{~F}$ \\
\hline 11. PMG & 8 & $\mathrm{~F}$ \\
\hline 12. PSO & 8.4 & $\mathrm{~F}$ \\
\hline 13. PCS & 8.3 & $\mathrm{M}$ \\
\hline 14. PPS & 8.6 & $\mathrm{~F}$ \\
\hline 15. VQC & 12.6 & $\mathrm{M}$ \\
\hline 16. VAP & 8.4 & $\mathrm{M}$ \\
\hline 17. WBC & 7.5 & $\mathrm{M}$ \\
\hline 18. APA & 11 & $\mathrm{~F}$ \\
\hline 19. CCV & 9.6 & $\mathrm{~F}$ \\
\hline
\end{tabular}

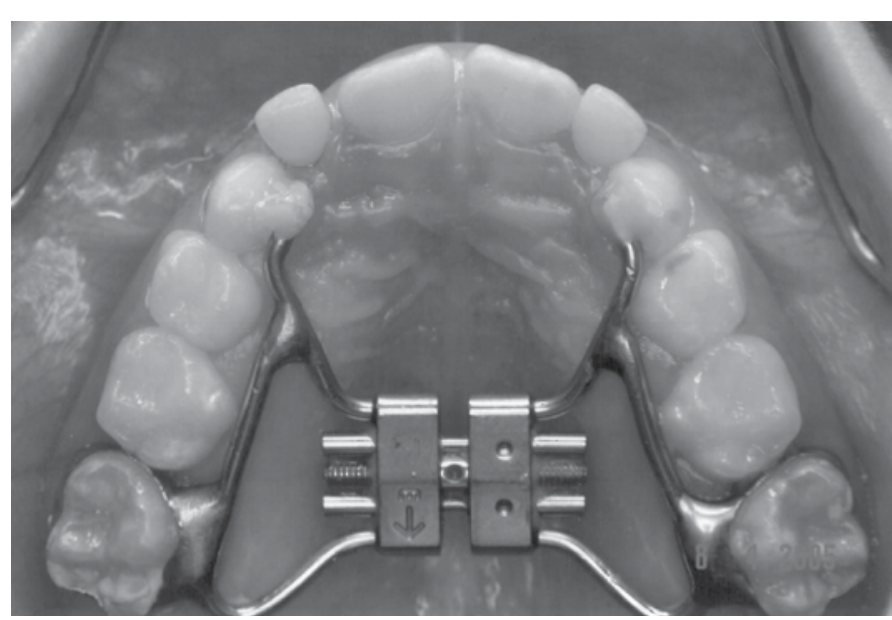

Figure 1. Biederman Modified appliance installed before RME.

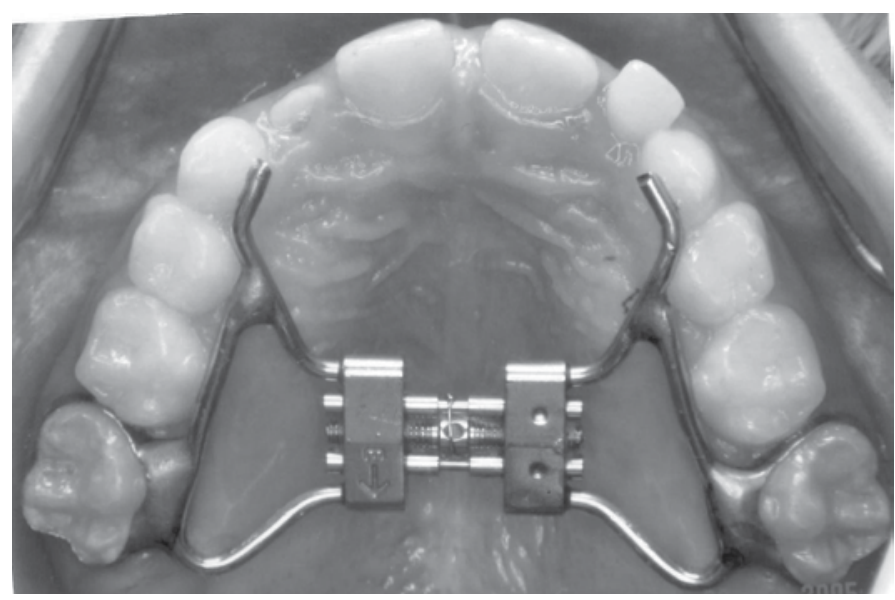

Figure 2. After RME with correction of the maxillary deficiency.

\section{MATERIALS AND METHODS}

This study was approved by the Research Ethics Committee of the Faculty of Dentistry of the University of São Paulo, under protocol 21/01.

19 patients were selected ( 9 females - $47.3 \%$ and 10 males $52.7 \%$ ), with a mean age of 8.9 years (Table 1 ). All the patients presented transversal maxillary deficiency and, based on the data obtained in the diagnosis, were submitted to rapid maxillary expansion in the Preventive Orthodontics Clinic of the Faculty of Dentistry of the University of Sáo Paulo, from January to December 2009. As inclusion criteria, patients could not have undergone previous orthodontic or otorhinolaryngologic treatment. For ethical reasons, it was not possible to evaluate a control group, for they would be subjected to unnecessary radiation, since they would not be treated orthodontically. In this way, we use data already published in the literature for comparison purposes with the selected sample.

The rapid maxillary expansion was performed using a modified Biederman dento-supported expander type. After a week of installation of the device, the activations were initiated, with one complete lap performed, and the patient responsible was instructed to activate $1 / 4$ turn every 12 hours. The activation time varied according to the need of expansion for correction of transverse maxillary deficiency (Figure 1 and 2).

All patients selected were submitted to a three-stage acoustic rhinometry test: before RME (M1), one week after the end of the activations (M2) and six months after the end of the activations (M3). The rhinometric examination was performed in each individual after it was acclimatized to the ambient conditions of the examination room, using RhinoScan apparatus from RhinoMetrics A/S - Denmark.

The procedure followed the determinations of the Standardization Committee of Acoustic Rhinometry. ${ }^{20}$ In each of the times, three measurements were performed in each nostril before and after the use of topical vasoconstrictor $(0.5 \mathrm{mg} / \mathrm{ml}$ - Pedrin Afrin) oxymetazoline hydrochloride. The measurements followed the following protocol: obtaining the measurements in the right nasal cavity and then in the left nasal cavity, both without the use of the vasoconstrictor. Thereafter, the application of three drops of topical vasoconstrictor was followed for a period of 10 to 15 minutes and the measurements were repeated following the same sequence.

Two area measurements were evaluated in each nostril (left and right): minimum cross-sectional area between $0 \mathrm{~mm}$ and $22 \mathrm{~mm}$ (MCA1), and minimum cross-sectional area between $22 \mathrm{~mm}$ and $54 \mathrm{~mm}$ (MCA2). Similarly, two volume measurements were evaluated on each side: Nasal space volume between 0 $\mathrm{mm}$ and $22 \mathrm{~mm}$ (VOL1) and Nasal space volume between 22 $\mathrm{mm}$ and $54 \mathrm{~mm}$ (VOL2). The results were reported in $\mathrm{cm}^{2}$ and $\mathrm{cm}^{3}$, respectively. The data were automatically calculated by the RhinoS $\operatorname{can}^{\oplus}$ software and were considered averages of the 3 measurements (Figure 3). 


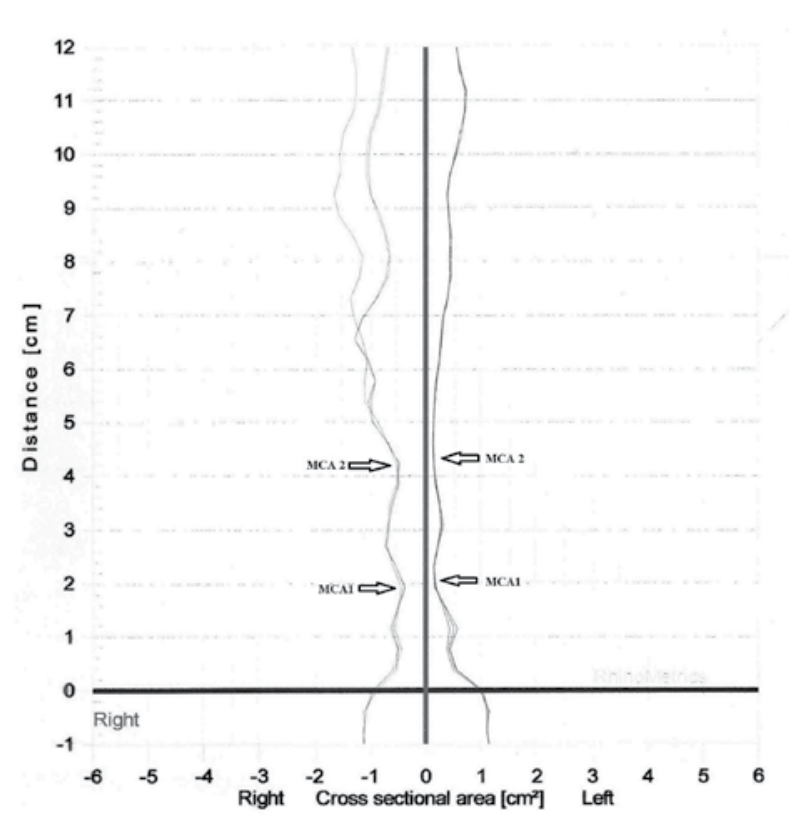

Figure 3. Graph automatically generated by RhinoScan ${ }^{\oplus}$ software with three curves obtained on each side. The central line representing the nasal septum and the curves representing the lateral walls of the nasal cavity on the right and left sides. From this graph the program provides the measures of MCA1 and MCA2 and volume 1 and 2.

\section{RESULTS}

In order to respond to the objectives of the study, first the measurements of nasal cavity area were recorded according to the use of vasoconstrictor, nasal cavity side and moment of the examination, using summary measures (mean, standard deviation). The results obtained were illustrated with the use of graphs of means with the respective standard errors. Subsequently, repeated measures (ANOVA) were performed with three factors, vasoconstrictor, nasal cavity side and time of the examination, followed by multiple Tukey comparisons to verify the level of significance between the mean measures found.

The tests were performed at a significance level of $5 \%$.

Table 2 shows that the MCA1 measurements do not vary between the right and left sides, with or without vasoconstrictor, but there seems to be a change between the moments of evaluation. The MCA2 measurement is lower without vasoconstrictor use and appears to increase in M2.

Table 3 shows that there was no mean change in MCA1 between the right and left sides ( $\mathrm{p}=0.299)$, between M1, M2 and M3 $(\mathrm{p}=0.122)$ or with vasoconstrictor use $(\mathrm{p}=0.510)$. But, for mean MCA2 there was statistically change between the moments $(\mathrm{p}=0.043)$ and with vasoconstrictor use $(\mathrm{p}=0.008)$ independent of the side.

Table 4 shows that the use of the vasoconstrictor increases the mean MCA2 ( $p=0.009)$ and the mean value of MCA2 is statistically higher in M2 when compared to M1 ( $\mathrm{p}=0.045)$.
Table 2. Description of MCA1 and MCA2 $\left(\mathrm{cm}^{2}\right)$ according to use of vasoconstrictor, right and left sides and moments M1, M2 and M3.

\begin{tabular}{|c|c|c|c|c|c|c|}
\hline \multirow[b]{2}{*}{ Vasoconstrictor } & \multirow[b]{2}{*}{ Slide } & \multirow[b]{2}{*}{ Moment } & \multicolumn{2}{|c|}{ MCA1 } & \multicolumn{2}{|c|}{ MCA2 } \\
\hline & & & Average & SD & Average & SD \\
\hline \multirow{6}{*}{$\begin{array}{c}\text { With } \\
\text { vasoconstrictor }\end{array}$} & \multirow{3}{*}{ Right } & M1 & 0.31 & 0.11 & 0.41 & 0.17 \\
\hline & & M2 & 0.36 & 0.10 & 0.50 & 0.15 \\
\hline & & M3 & 0.34 & 0.11 & 0.44 & 0.16 \\
\hline & \multirow{3}{*}{ Left } & M1 & 0.38 & 0.47 & 0.43 & 0.16 \\
\hline & & M2 & 0.30 & 0.11 & 0.47 & 0.18 \\
\hline & & M3 & 0.25 & 0.10 & 0.38 & 0.17 \\
\hline \multirow{6}{*}{$\begin{array}{c}\text { No } \\
\text { vasoconstrictor }\end{array}$} & \multirow{3}{*}{ Right } & M1 & 0.30 & 0.12 & 0.36 & 0.15 \\
\hline & & M2 & 0.35 & 0.09 & 0.43 & 0.14 \\
\hline & & M3 & 0.31 & 0.13 & 0.42 & 0.29 \\
\hline & \multirow{3}{*}{ Left } & M1 & 0.25 & 0.11 & 0.32 & 0.14 \\
\hline & & M2 & 0.40 & 0.47 & 0.38 & 0.13 \\
\hline & & M3 & 0.24 & 0.10 & 0.35 & 0.24 \\
\hline
\end{tabular}

Table 3. Results of ANOVA for MCA1 and MCA2 measurement. $\left({ }^{*}\right.$ statistically significant $)$.

\begin{tabular}{ccccccc}
\hline & & \multicolumn{2}{c}{ MCA1 } & \multicolumn{2}{c}{ MCA2 } \\
\hline Factor & fd num. & fd den. & Value F & P & Value F & P \\
\hline $\begin{array}{c}\text { Vaso } \\
\text { constrictor }\end{array}$ & 1 & 18 & 0.45 & 0.510 & 8.75 & $0.008^{*}$ \\
\hline Side & 1 & 18 & 1.14 & 0.299 & 3.01 & 0.100 \\
\hline Moment & 2 & 36 & 2.23 & 0.122 & 3.44 & $0.043^{*}$ \\
\hline Vaso*Side & 1 & 18 & 0.01 & 0.935 & 0.39 & 0.538 \\
\hline Vaso*Moment & 2 & 36 & 1.70 & 0.198 & 0.63 & 0.540 \\
\hline Side*Moment & 2 & 36 & 1.12 & 0.337 & 0.74 & 0.482 \\
\hline $\begin{array}{l}\text { Vaso*Side } \\
\text { *Moment }\end{array}$ & 2 & 36 & 1.68 & 0.201 & 0.14 & 0.869 \\
\hline
\end{tabular}

Table 4. Results of Tukey's multiple comparisons between moments M1, M2 and $M 3$ and use of vasoconstrictor in MCA2. ( ${ }^{*}$ statistically significant) WV - With vasoconstrictor / NV - No vasoconstrictor.

\begin{tabular}{ccccccc}
\hline Factor & $\begin{array}{c}\text { Compa- } \\
\text { ration }\end{array}$ & Estimate & $\begin{array}{c}\text { Standard } \\
\text { error }\end{array}$ & $\begin{array}{c}\text { Value } \\
\mathbf{T}\end{array}$ & $\mathbf{f d}$ & $\mathbf{p}$ \\
\hline $\begin{array}{c}\text { Vaso } \\
\text { constrictor }\end{array}$ & WV-NV & 0.063 & 0.022 & 2.91 & 18 & $0.009^{*}$ \\
\hline \multirow{2}{*}{ Moment } & M1-M2 & -0.066 & 0.026 & -2.49 & 36 & $0.045^{*}$ \\
\cline { 2 - 7 } & M1-M3 & -0.017 & 0.026 & -0.64 & 36 & 0.798 \\
\cline { 2 - 7 } & M2-M3 & 0.049 & 0.026 & 1.84 & 36 & 0.170 \\
\hline
\end{tabular}


Table 5 suggests that the measure of VOL 1 is smaller on the left side than on the right side. The VOL 2 is lower without vasoconstrictor use and there is little variation of this measure between the evaluated moments.

Table 5. Description of VOL1 and VOL2 according to the use of vasoconstrictor, right and left sides and moments M1, M2 and M3.

\begin{tabular}{|c|c|c|c|c|c|c|}
\hline \multirow[b]{2}{*}{ Vasoconstrictor } & \multirow[b]{2}{*}{ Slide } & \multirow[b]{2}{*}{ Moment } & \multicolumn{2}{|c|}{ VOL1 } & \multicolumn{2}{|c|}{ VOL2 } \\
\hline & & & Average & SD & Average & SD \\
\hline \multirow{6}{*}{$\begin{array}{c}\text { With } \\
\text { vasoconstrictor }\end{array}$} & \multirow{3}{*}{ Right } & M1 & 1.15 & 0.18 & 3.14 & 3.14 \\
\hline & & M2 & 1.23 & 0.19 & 3.18 & 3.18 \\
\hline & & M3 & 1.22 & 0.20 & 3.43 & 3.43 \\
\hline & \multirow{3}{*}{ Left } & M1 & 1.06 & 0.15 & 3.27 & 3.27 \\
\hline & & M2 & 1.17 & 0.34 & 3.37 & 3.37 \\
\hline & & M3 & 1.01 & 0.29 & 2.87 & 2.87 \\
\hline \multirow{6}{*}{$\begin{array}{c}\text { No } \\
\text { vasoconstrictor }\end{array}$} & \multirow{3}{*}{ Right } & M1 & 1.13 & 0.28 & 2.43 & 2.43 \\
\hline & & M2 & 1.22 & 0.17 & 2.69 & 2.69 \\
\hline & & M3 & 1.23 & 0.20 & 2.36 & 2.36 \\
\hline & \multirow{3}{*}{ Left } & M1 & 1.07 & 0.23 & 2.32 & 2.32 \\
\hline & & M2 & 1.10 & 0.25 & 2.70 & 2.70 \\
\hline & & M3 & 1.09 & 0.25 & 2.15 & 2.15 \\
\hline
\end{tabular}

Table 6 shows that VOL 1 varies between the right and left sides $(\mathrm{p}<0.001)$ and between moments M1, M2 and M3 ( $\mathrm{p}=0.028)$, regardless of whether or not vasoconstrictor is used. For VOL 2 we observe a statistically significant increase only for the use of the vasoconstrictor $(\mathrm{p}<0.001)$ independent of the side or the moment of evaluation and, therefore, Tukey's multiple comparisons test was not performed for this measure.

Table 6. Result of ANOVA for measurement of VOL1 and VOL2. $\left({ }^{*}\right.$ statistically significant $)$.

\begin{tabular}{ccccccc}
\hline & & \multicolumn{2}{c}{ VOL1 } & \multicolumn{2}{c}{ VOL2 } \\
\hline Factor & fd num. & fd den. & Value F & P & Value F & P \\
\hline $\begin{array}{c}\text { Vaso } \\
\text { constrictor }\end{array}$ & 1 & 18 & 0.00 & 0.963 & 44.56 & $<0.001^{*}$ \\
\hline Side & 1 & 18 & 25.66 & $<0.001^{*}$ & 0.63 & 0.439 \\
\hline Moment & 2 & 36 & 3.94 & $0.028^{*}$ & 2.06 & 0.142 \\
\hline Vaso*Side & 1 & 18 & 0.21 & 0.652 & 0.01 & 0.931 \\
\hline $\begin{array}{c}\text { Vaso*Moment } \\
\text { Side*Moment }\end{array}$ & 2 & 36 & 1.24 & 0.302 & 0.71 & 0.499 \\
\hline $\begin{array}{c}\text { Vaso*Side } \\
\text { *Moment }\end{array}$ & 2 & 36 & 1.80 & 0.180 & 1.68 & 0.200 \\
\hline
\end{tabular}

In Table 7 it can be seen that the mean VOL1 is statistically higher on the right side $(\mathrm{p}<0.001)$ and suffers a mean increase in M2 compared to M1 ( $\mathrm{p}=0.025)$ and continues higher in M3 $(\mathrm{p}=0.271)$.

Table 7. Results of Tukey multiple comparisons between right and left sides and moments M1, M2 and M3 for the measurement of VOL 1. ( ${ }^{*}$ statistically significant).

\begin{tabular}{ccccccc}
\hline \multirow{2}{*}{ Factor } & $\begin{array}{c}\text { Compa- } \\
\text { ration }\end{array}$ & Estimate & $\begin{array}{c}\text { Standard } \\
\text { error }\end{array}$ & $\begin{array}{c}\text { Value } \\
\mathbf{T}\end{array}$ & $\mathbf{f d}$ & $\mathbf{p}$ \\
\hline \multirow{2}{*}{ Side } & Right-Left & 0.114 & 0.023 & 4.97 & 18 & $<0.001^{*}$ \\
\cline { 2 - 7 } Moment & M1-M2 & -0.077 & 0.028 & -2.75 & 36 & $0.025^{*}$ \\
\cline { 2 - 7 } & M1-M3 & -0.033 & 0.028 & -1.17 & 36 & 0.476 \\
\cline { 2 - 7 } & M2-M3 & 0.044 & 0.028 & 1.57 & 36 & 0.271 \\
\hline
\end{tabular}

\section{DISCUSSION}

According to the literature, according to the literature, many studies attempt to evaluate the relationship between rapid maxillary expansion and increased nasal cavity size by different methods. ${ }^{26}$ The purpose of this study was to evaluate the nasal cavity before and after RME using acoustic rhinometry, which provides Minimal crossection areas and nasal volumes. When analyzing the results, we can observe in Table 2 the variation of the mean values of MCA1 and MCA2 between moments M1, $\mathrm{M} 2$ and M3, with their respective standard errors of the right and left sides. In MCA1 for the left side, without the use of vasoconstrictor, the MCA1 ranged from $0.25 \mathrm{~cm}^{2}$ in $\mathrm{M} 1$ to 0.40 $\mathrm{cm}^{2}$ in M2, but there was a recurrence in $\mathrm{M} 3$ to $0.24 \mathrm{~cm}^{2}$. For the right side, without the use of vasoconstrictor we observe a variation of the same form, being that, in M1, the average value of MCA1 was $0,30 \mathrm{~cm}^{2}$, in $\mathrm{M} 20,35 \mathrm{~cm}^{2}$ and in $M 30,31 \mathrm{~cm}^{2}$. However, this observed increase was not statistically significant, possibly by sample size (Table 3).

Similar results were found by Enoki et al. ${ }^{18}$ who investigated ${ }^{29}$ patients undergoing RME and observed a significant increase of MCA1 immediately after RME. The authors found that in M1 (Pre-RME) the mean MCA1 of the left and right nasal cavities was $0.987 \mathrm{~cm}^{2}$. After RME, this measure increased to 1.006 $\mathrm{cm}^{2}$ and after the use of restraint, these authors also observed a recurrence of this measure to $0.973 \mathrm{~cm}^{2}$.

Bicakci et al. ${ }^{27}$ reported an increase of $8.7 \%$ in MCA1 in a group of patients treated with RME between M1 (Pre-RME) and M3 (Post-retention). The authors also observed that there was a recurrence of $6.3 \%$ between M2 (Post-RME) and M3.

As shown in Table 3, in this study no difference in behavior was observed between the right and left sides of the nasal cavity, similarly to the study of Cappellette et al. ${ }^{12}$, however, these authors observed a statistically significant increase in MCA1 immediately after RME. 
De Fellippe et al. ${ }^{16}$ observed increased values in MCA1 after RME. In the pre-RME phase (M1) in the left nasal cavity, they found MCA1 of $0.38 \mathrm{~cm}^{2}$, in the post-RME phase (M2) increased to $0.48 \mathrm{~cm}^{2}$, three months after RME (M3) the measurement was $0.47 \mathrm{~cm}^{2}$ and nine months after RME (M4) was $0.51 \mathrm{~cm}^{2}$. For the right nasal cavity, the behavior was similar, with values of MCA1 in $M=0.41 \mathrm{~cm}^{2}, \mathrm{M} 2=0.48 \mathrm{~cm}^{2}$, $\mathrm{M} 3=0.48 \mathrm{~cm}^{2}$ and $\mathrm{M} 4=0.53 \mathrm{~cm}^{2}$. Statistical significance was found between M1 and M2, M1 and M3 and M1 and M4, but between the moments M2 and M3 there was no change with statistical significance that, according to the authors, represented a moment of stability after removing the appliance.

Tables 2 and 3 show the MCA2 values and it is noted that there was a change in the measurements between the evaluated moments. For the left nasal cavity in $\mathrm{M} 1$ we found $0.36 \mathrm{~cm}^{2}$, in M2 $0,43 \mathrm{~cm}^{2}$ and in M3 $0,42 \mathrm{~cm}^{2}$. For the right nasal cavity the value found in $\mathrm{M} 1$ was $0.32 \mathrm{~cm}^{2}$, in $\mathrm{M} 20,38 \mathrm{~cm}^{2}$ and in M3 $0,35 \mathrm{~cm}^{2}$. After the statistical analysis (Table 3) we noticed that the observed increase was significant in M2 when compared to M1, however, between M2 and M3 no significant change was found, which can be analyzed as a moment of stability as stated by De Fellippe et al. ${ }^{16}$

The results found by Enoki et al. ${ }^{18}$ show variation of the MCA2 values of the right and left nasal cavities. In M1 (Pre-RME) the value found was $0.732 \mathrm{~cm}^{2}$, in M2 (Post-RME) $0.780 \mathrm{~cm}^{2}$ and in M3 (3 months post-retainer) $0.763 \mathrm{~cm}^{2}$. However, none of the changes presented statistical significance. Cappellette et al. ${ }^{12}$ reported a statistically significant increase in MCA2 immediately after RME.

Our results show that when MCA2 means between M1 e M3 is compared, there is no statistically significant difference. Showing that after the period of containment there is a tendency of relapse to the initial measurements.

Regarding the nasal volume, it can be observed in Tables 5, 6 and 7 that the mean VOL1 increased between M1 and M2 $(p=0.025)$, values statistically significant for the right nasal cavity. The mean variation was $1.13 \mathrm{~cm}^{3}$ in $\mathrm{M} 1$, to $1.22 \mathrm{~cm}^{3}$ in $\mathrm{M} 2$, remaining stable in $\mathrm{M} 3\left(1.23 \mathrm{~cm}^{3}\right)$. In the left nasal cavity there was no significant variation in the moments of evaluation. Already, Cappelletteet al. ${ }^{12}$ reported an increase in VOL 1, after RME, on both sides of the nasal cavity. The mean increase was $0.07 \mathrm{~cm}^{3}$ for both right and left the nasal cavity.

De Fellippe et al. ${ }^{15}$ also observed increased nasal cavity volume after RME. The increase between M1-M2, M1-M3 and M1M4 moments were significant, but no significant change was observed between M2-M3 and M3-M4. These results, in the opinion of the authors, demonstrate the stability of the volume increase achieved. Likewise, Babacan et al. ${ }^{28}$ observed a statistically significant increase of $14.48 \%$ in the nasal volume between M1 (Pre-RME) and M2 (post-RME).

For the measurements of nasal volume 2, we observed that there was an increase of $0.26 \mathrm{~cm}^{3}$ in the right nasal cavity between moments M1 and M2, without statistical significance. On the left side, an increase of $0.38 \mathrm{~cm}^{3}$ was observed between the moments M1 and M2, which were not statistically significant (Tables 5 and 6). Similarly, Cappellette et al. ${ }^{12}$ reported an increase, between $\mathrm{M} 1$ and $\mathrm{M} 2$, of $0.34 \mathrm{~cm}^{3}$, statistically significant $(\mathrm{p}=0.023)$, from VOL 2 to the right nasal cavity, and on the left side the increase between these moments of evaluation was $0.21 \mathrm{~cm}^{3}$, not statistically significant.

\section{CONCLUSION}

The results found in this study allow us to affirm that RME increases significantly the anterior region of the nasal cavity immediately to the procedure, however, after the period of containment there is a tendency of recurrence of this increase returning to values close to the initial cross-sectional area of the nasal cavity. Therefore, the indication of this procedure should be performed only for correction of dentoalveolar problems and no expectation of correction of respiratory function should be generated.

\section{CONFLICT OF INTEREST}

The authors declare no conflict of interest. 


\section{REFERENCES}

1. Zavras Al, White GE, Rich A, Jackson AC. Acoustic rhinometry in the evaluation of children with nasal or oral respiration. J Clin Pediatr Dent 1994;18(3):203-10

2. Linder-Aronson $S$, Backstrom A. Respiratory function in relation to facial morphology and the dentition. Br J Orthod 1979;6(2):59-71.

3. McNamara Junior JA. Influence of respiratory pattern on craniofacial growth. Angle Orthod 1981;51(4):269-300.

4. O'ryan FS, Gallagher DM, LaBanc JP, Epker BN. The relation between nasorespiratory function and dentofacial morphology: a review. Am J Orthod 1982;82(5):403-10.

5. Diamond O. Tonsil and adenoids: why the dilemma? Am J Orthod 1980;78(5):495-503.

6. Hartsook JT. Mouth breathing as a primary etiologic factor in the production of malocclusion. J Dent Child 1946;13(4):91-4.

7. Angell EH. Treatment of irregularity of the permanent adult teeth. Part I. Dental Cosmos 1860 1:540-4.

8. Hartgerink DV, Vig PS, Abbott DW. The effects of rapid maxillary expansion on nasal airway resistance. Am J Orthod Dentofac Orthop1987;92(5):381-9.

9. Haas AJ. Rapid expansion of the maxillary dental arch and nasal cavity by opening the midpalatal suture. Angle Orthod 1961;31:73-90.

10. Wertz RA. Changes in nasal airflow incident to rapid maxillary expansion. Angle Orthod 1968;38(1):1-11.

11. Warren DW, Hershey HG, Turvey TA, Hinton VA, Hairfield WM. The nasal airway following maxillary expansion. Am J Orthod Dentofacial Orthop. 1987;91(2):111-6.

12. Cappellette Júnior M, Cruz OLM, Carlini D, Weckx LL, Pignatari SS. Evaluation of nasal capacity before and after rapid maxillary expansion. Am J Rhinol 2008;22(1):74-7.

13. Baratieri C, Alves M, de Souza MM G, de Souza Araùjo MT, Cople Maia L. Does rapid maxillary expansion have long-term effects on airway dimensions and breathing? Am J Orthod Dentofacial Orthop 2011;140(2):146-56.

14. Ribeiro ANC, de Paiva JB, Rino-Neto J, Illipronti-Filho E, Mongelli FS. Upper airway expansion after rapid maxillary expansion evaluated with cone beam computed tomography. Angle Orthod 2012;82(3):458-63.

15. De Fellippe NLO, Da Silveira AC, Viana G, Kusnoto B, Smith B, Evans CA. Relationship between rapid maxillary expansion and nasal cavity size and airway resistance: short and long term effects. Am J Orthod Dentofac Orthop 2008;134(3):370-82.
16. De Felippe NLO, Bhushan N, Da Silveira AC, Viana G, Smith B. Longterm effects of orthodontic therapy on the maxillary dental arch and nasal cavity. Am J Orthod Dentofacial Orthop. 2009;136(4):490e1-e8.

17. McNamara Jr JA, Lione R, Franchi L, Angelieri F, Cevidanes LH, Darendeliler MA, Cozza P. The role of rapid maxillary expansion in the promotion of oral and general health. Progress in Orthodontics 2015;16:33-9.

18. Enoki C, Valera FCP, Lessa FCR, Elias AM, Matsumoto MAN, AnselmoLima WT. Effect of rapid maxillary expansion on the dimension of the nasal cavity and on nasal air resistance. Int J Pediatr Otorhinolaryngol. 2006;70(7):1225-30

19. Gordon JM, Rosenblatt M, Witmans M, Carey JP, Heo G, Major PW, Flores-Mir C. Rapid Palatal Expansion Effects on Nasal Airway Dimensions as Measured by Acoustic Rhinometry. Angle Orthod 2009;79(5):1000-7.

20. Hilberg O, Jackson AC, Swift DL, Pedersen OF. Acoustic rhinometry: evaluation of nasal cavity geometry by acoustic reflection. J Appl Physiol 1989;66(1):295-303.

21. Corey JP. Acoustic Rhinometry: should we be using it? Curr Opin Otolaryngol Head Neck Surg 2006;14(1):29-34.

22. Haavisto LE, Sipilä JI. Acoustic rhinometry in children: Some practical aspects and influence of age and body surface area on results. Am J Rhinol 2008;22(4):416-9.

23. Gilain L, Coste A, Ricolfi F, Dahan E, Marliac D Peynegre R, Harf A, Louis B. Nasal cavity geometry measured by acoustic rhinometry and computed tomography. Arch Otolaryngol Head Neck Surg 1997;123(4):401-5.

24. Szucs E, Clemente PA. Acoustic rhinometry and rhinomanometry in the evaluation of nasal patency of patients with nasal septal deviation. Am J Rhinol 1998;12(5):345-52.

25. Tsolakis IA, Venkat D, Hans MG, Alonso A, Palomo JM. When static meets dynamic: Comparing cone-beam computed tomography and acoustic reflection for upper airway analysis. Am J Orthod Dentofacial Orthop 2016;150(4):643-50.

26. Lee WC, Tu YK, Huang CS, Chen R, Fu MW, Fu E. Pharyngeal airway changes following maxillary expansion or protraction: A meta-analysis. Orthod Craniofac Res 2018;21(1):4-11.

27. Bicakci AA, Agar U, Sökücü O, Babacan H, Doruk C. Nasal airway changes due to rapid maxillary expansion timing. Angle Orthod. 2005;75(1):1-6.

28. Babacan H, Sokuco O, Doruk C, Ay S. Rapid maxillary expansion and surgically assisted rapid maxillary expansion effects on nasal volume. Angle Orthod 2006;76(1):66-71. 